NONCOMMUTATIVE GEOMETRY

AND QUANTUM GROUPS

BANACH CENTER PUBLICATIONS, VOLUME 61

INSTITUTE OF MATHEMATICS

POLISH ACADEMY OF SCIENCES

WARSZAWA 2003

\title{
COMMUTATOR REPRESENTATIONS OF COVARIANT DIFFERENTIAL CALCULI
}

\author{
KONRAD SCHMÜDGEN \\ Fakultät für Mathematik und Informatik, Universität Leipzig \\ Augustusplatz 10, 04109 Leipzig, Germany \\ E-mail: schmuedg@mathematik.uni-leipzig.de
}

1. Introduction. A central role in Alain Connes' noncommutative geometry $[\mathrm{C}]$ is played by the notion of a spectral triple. Recall that a spectral triple $(\mathcal{A}, \mathcal{H}, D)$ is a triple of a $*$-algebra $\mathcal{A}$, a $*$-representation $\rho$ of $\mathcal{A}$ by bounded operators on a Hilbert space $\mathcal{H}$ and a possibly unbounded self-adjoint operator $D$ on $\mathcal{H}$ with compact resolvent such that

the commutator $[D, \rho(a)]$ is bounded for all $a \in \mathcal{A}$.

The standard example of a spectral triple is given by the Dirac operator $D$ of a compact spin manifold $M$. In this example $\mathcal{A}$ is the *-algebra of $C^{\infty}$-functions on $M, \mathcal{H}$ is the Hilbert space of square-integrable sections of the spinor bundle on $M$ and $\rho$ is given by the pointwise multiplication of sections.

On the other hand, there is a theory of covariant differential calculi on quantum groups. It began by the poineering work of S.L. Woronowicz $[\mathrm{W}]$ and contains a number of interesting results developed since then (see e.g. [KS], Chapter 14, for a thorough treatment). This theory fits nicely into important structures of quantum groups such as $L$-functionals ([KS], 10.1.3), Hopf bimodules, locally finite parts of adjoint actions of Drinfeld-Jimbo algebras [JL] and others. For the standard quantum groups $G L_{q}(n), S L_{q}(n), O_{q}(n), S p_{q}(2 n)$ there exist some distinguished bicovariant differential calculi ([KS], 14.6). Thus, it is quite natural to look for the relations between this theory and Connes' noncommutative geometry. In [S1] it was shown that the $3 D$-calculus and the $4 D_{ \pm}$-calculi on the quantum group $S U_{q}(2)$ cannot be described by spectral triples. More precisely, it is not difficult to see that the corresponding commutation relations between forms and functions cannot be given by bounded commutators as required in condition $(*)$ above. But there are plenty of realizations of these calculi by means of unbounded commutators. Such realizations are the theme of the present article.

2000 Mathematics Subject Classification: 17B37, 46L87, 81R50.

Key words and phrases: quantum groups, noncommutative geometry.

The paper is in final form and no version of it will be published elsewhere. 
Let us return for a moment to the spectral triple of the Dirac operator $D$ on a compact spin manifold $M$. In this case there are two important additional facts: First, the operator $D$ is covariant with respect to the symmetry group acting on the manifold and secondly, the commutator with the Dirac operator gives the ordinary first order differential calculus of the manifold. In our approach we put our emphasis on these two properties. That is, we begin with a distinguished covariant first order differential calculus on a quantum group and look for a "nice" commutator representation of the calculus. This will be done in Sections 3 and 4 where the cases of left-covariant calculi and of bicovariant calculi are treated separately. These sections are taken from the paper [S2] where proofs of all unproven results can be found.

In Sections 5 and 6 we construct Dirac operators for covariant differential calculi on two simple non-compact quantum spaces (quantum disc and real quantum quarter plane). By means of an appropriate Hilbert space representation of the corresponding coordinate algebra $\mathcal{O}$ we first develop an algebra $\mathcal{O}_{c}$ of "integrable $C^{\infty}$-functions" and an "invariant integration" on the quantum space. The covariant differential calculus is given on the coordinate algebra $\mathcal{O}$. Using a commutator representation of the calculus in Hilbert space it is easily extended to a calculus $\Gamma^{\wedge}$ of the larger algebra $\mathcal{O}+\mathcal{O}_{c}$. Then we define a covariant metric on $\Gamma^{\wedge}$, a covariant scalar product on the restriction $\Gamma_{c}^{\wedge}$ of $\Gamma^{\wedge}$ to the subalgebra $\mathcal{O}_{c}$ and finally a Dirac operator in the corresponding Hilbert space. For the quantum disc (Section 5) this was done in the paper [SSV]. The treatment of the real quantum quarter plane in Section 6 is based on the paper [S3], where more results and details can be found. We are convinced that a similar procedure works for other covariant differential calculi on non-compact quantum spaces as well.

Let us mention some other recent papers dealing with related topics: Dirac operators for bicovariant differential calculi on quantum groups are constructed in [H1], twisted cyclic cocycles for quantum groups are studied in [KMT], and covariant spectral triples are investigated in [PS].

We freely use the notation and the results on Hopf algebras and on covariant differential calculi from the monograph [KS].

2. Some basic definitions. Let $\mathcal{X}$ be a unital associative complex algebra. A first order differential calculus (abbreviated, FODC) of $\mathcal{X}$ is an $\mathcal{X}$-bimodule $\Gamma$ with a linear mapping $d: \mathcal{X} \rightarrow \Gamma$ such that $d(x y)=d x \cdot y+x \cdot d y$ for $x, y \in \mathcal{X}$ and $\Gamma$ is the linear span of $x \cdot d y$, where $x, y \in \mathcal{X}$.

A differential calculus (abbreviated, DC) of $\mathcal{X}$ is a graded algebra $\Gamma^{\wedge}=\bigoplus_{n=0}^{\infty} \Gamma^{\wedge n}$, where $\Gamma^{\wedge 0}=\mathcal{X}$, with a linear mapping $d: \Gamma^{\wedge} \rightarrow \Gamma^{\wedge}$ of degree one such that $d^{2}=$ $0, d(\eta \wedge \varphi)=d \eta \wedge \varphi+(-1)^{n} \eta \wedge d \varphi$ for $\eta \in \Gamma^{\wedge n}, \varphi \in \Gamma^{\wedge}$ and $\Gamma^{\wedge n}$ is the linear span of $x_{0} d x_{1} \wedge \ldots \wedge d x_{n}$, where $x_{0}, x_{1}, \ldots, x_{n} \in \mathcal{X}$.

An algebraic commutator representation of a first order calculus $\Gamma$ of $\mathcal{X}$ is a pair $(C, \rho)$ of an element $C$ of an algebra $\mathcal{Y}$ and an algebra homomorphism $\rho: \mathcal{X} \rightarrow \mathcal{Y}$ such that there exists a linear mapping $\tau: \Gamma \rightarrow \mathcal{Y}$ satisfying

$$
\tau(x d y)=i \rho(x)(C \rho(y)-\rho(y) C), \quad x, y \in \mathcal{X} .
$$


Now let $\mathcal{X}$ be a $*$-algebra. An FODC $\Gamma$ of $\mathcal{X}$ is called a $*$-calculus if there exists a vector space involution $\eta \rightarrow \eta^{*}$ of $\Gamma$ such that $(x \cdot d y \cdot z)^{*}=z^{*} \cdot d\left(y^{*}\right) \cdot x^{*}$ for $x, y, z \in \mathcal{X}$.

Let $\mathcal{D}$ be a dense linear subspace of a Hilbert space and let $L(\mathcal{D})$ be the algebra of linear operators mapping $\mathcal{D}$ into itself. A pair $(C, \rho)$ of a symmetric operator $C \in L(\mathcal{D})$ and a $*$-representation $\rho$ of $\mathcal{X}$ on $\mathcal{D}$ is called a commutator representation of a $*$-calculus $\Gamma$ if $(C, \rho)$ is an algebraic commutator representation of $\Gamma$ with $\mathcal{Y}=L(\mathcal{D})$.

3. Left-covariant first order differential calculi on Hopf algebras. Throughout this section $\mathcal{A}$ is a Hopf algebra. We denote by $\mathcal{A}^{\circ}$ the Hopf dual of $\mathcal{A}$ and use the Sweedler notation $\Delta(a)=a_{(1)} \otimes a_{(2)}$ for the comultiplication $\Delta(a)$ of $a \in \mathcal{A}$. The counit of $\mathcal{A}$ is denoted by $\varepsilon$.

Let $\Gamma$ be a left-covariant FODC on $\mathcal{A}$ such that the vector space $\Gamma_{\text {inv }}$ of left-invariant elements of $\Gamma$ is finite dimensional. Fix a basis $\left\{\omega_{1}, \ldots, \omega_{n}\right\}$ of $\Gamma_{\text {inv }}$. Then there exist linear functionals $X_{k}, f_{k}^{j} \in \mathcal{A}^{\circ}, j, k=1, \ldots, n$, such that

$$
\begin{aligned}
& d a=\sum_{k} a_{(1)} X_{k}\left(a_{(2)}\right) \omega_{k} \equiv \sum_{k} \partial_{X_{k}}(a) \omega_{k}, \quad a \in \mathcal{A}, \\
& \Delta\left(X_{k}\right)=\varepsilon \otimes X_{k}+\sum_{j} X_{j} \otimes f_{k}^{j} .
\end{aligned}
$$

The linear span $\mathcal{T}_{\Gamma}$ of functionals $X_{1}, \ldots, X_{n}$ is called the quantum tangent space of $\Gamma$. The next proposition characterizes left-covariant FODC in terms of their quantum tangent spaces.

Proposition 1. (i) A finite dimensional vector space $\mathcal{T}$ of $\mathcal{A}^{\circ}$ is the quantum tangent space of a left-covariant $F O D C \Gamma$ of $\mathcal{A}$ iff $X(1)=0$ for $X \in \mathcal{T}$ and $\mathcal{T} \oplus \mathbb{C} \cdot \varepsilon$ is a right coideal of $\mathcal{A}^{\circ}$ (i.e. $\Delta(X)-\varepsilon \otimes X \in \mathcal{T} \otimes \mathcal{A}^{\circ}$ for $X \in \mathcal{T}$ ).

(ii) Suppose that $\mathcal{A}^{\circ}$ separates the point of $\mathcal{A}$. Then the left-covariant FODC $\Gamma$ from (i) is bicovariant iff $\operatorname{ad}_{R}(f) X \equiv S\left(f_{(1)}\right) X f_{(2)} \in \mathcal{T}$ for all $X \in \mathcal{T}$ and $f \in \mathcal{A}^{\circ}$.

Proof. [KS], Proposition 14.6 and Corollary 14.9.

For quantum groups there is no canonical differential calculus as in classical differential geometry. Thus it is natural to ask: How many 3-dimensional left-covariant differential calculi do exist on the Hopf algebra $\mathcal{O}\left(S L_{q}(2)\right)$ ?

This problem was investigated by I. Heckenberger [H2]. He found all left-covariant FODC $\Gamma$ for which the left-invariant one-forms associated with the elements $u_{2}^{1}, u_{1}^{2}, u_{1}^{1}-$ $u_{2}^{2}$ form a left module basis of $\Gamma$ and the universal higher order calculi have the same dimensions as in the classical case. I. Heckenberger showed that for $q$ not a root of unity there are precisely 11 FODC on $\mathcal{O}\left(S L_{q}(2)\right)$ satisfying the above and two additional reasonable assumptions. The quantum tangent spaces of some of these calculi are:

$$
\begin{aligned}
& \mathcal{T}_{1}: E K, F K, \varepsilon-K^{2} \quad(3 \mathrm{D} \text {-calculus of Woronowicz }), \\
& \mathcal{T}_{2}: E K^{-1}, F K^{-1}, \varepsilon-K^{2}, \\
& \mathcal{T}_{3}: E K, F K^{-3}, \varepsilon-K^{-4} \\
& \mathcal{T}_{4}: E K, F K^{5}, \varepsilon-K^{4} \\
& \mathcal{T}_{5}: E K^{-3}, F K^{-3}, \varepsilon-K^{-4},
\end{aligned}
$$




$$
\begin{aligned}
& \mathcal{T}_{6}: E K^{5}, F K, \varepsilon-K^{4}, \\
& \mathcal{T}_{7}: E K^{-3}, F K, \varepsilon-K^{-4},
\end{aligned}
$$

where $E, F, K, K^{-1}$ are the generators of the quantized enveloping algebra $\breve{U}_{q}\left(s l_{2}\right.$ ) (in the notation of $[\mathrm{KS}]$, p. 57). One easily checks that the vector spaces $\mathcal{T}_{1}, \ldots, \mathcal{I}_{7}$ satisfy the conditions of Proposition 1(i), so they define indeed left-covariant FODC on $\mathcal{O}\left(S L_{q}(2)\right)$.

None of these 3-dimensional left-covariant FODC is bicovariant. If $q$ is not a root of unity, then there precisely two bicovariant FODC $\Gamma$ on $\mathcal{O}\left(S L_{q}(2)\right)$ such that $\operatorname{dim} \Gamma_{\text {inv }}>1$ and the differentials $d u_{j}^{i}, i, j=1,2$, of the matrix entries generate $\Gamma$ as a left module. These are the $4 D_{ \pm}$-calculi $\Gamma_{ \pm}$of Woronowicz. Their quantum tangent spaces are:

$$
\mathcal{T}_{ \pm}: \varepsilon_{ \pm} E K^{-1}, \varepsilon_{ \pm} F K^{-1}, \varepsilon_{ \pm} K^{2}+\lambda^{2} q^{-1} \varepsilon_{ \pm} F E-\varepsilon, \varepsilon_{ \pm} K^{-2}-\varepsilon,
$$

where $\lambda:=q-q^{-1}, \varepsilon_{+}:=\varepsilon$ and $\varepsilon_{-}$is the character defined by $\varepsilon_{-}\left(u_{1}^{1}\right)=\varepsilon_{-}\left(u_{2}^{2}\right)=-1$ and $\varepsilon_{-}\left(u_{2}^{1}\right)=\varepsilon_{-}\left(u_{1}^{2}\right)=0$.

The FODC $\Gamma_{j}$ with quantum tangent spaces $\mathcal{T}_{j}, j=1,2,5,+,-$, are $*$-calculi of the Hopf *-algebra $\mathcal{O}\left(S U_{q}(2)\right)$. None of these *-calculi has a non-trivial commutator representation with bounded commutators. For $\mathcal{T}_{1}, \mathcal{T}_{2}, \mathcal{T}_{+}$and $\mathcal{T}_{-}$this was proved in [S1]; the proof for $\mathcal{T}_{5}$ is similar.

Let us return to an arbitrary finite dimensional FODC $\Gamma$ of a Hopf algebra $\mathcal{A}$. We shall construct a faithful algebraic commutator representation of $\Gamma$.

For this we essentially need the cross product algebra $\mathcal{A} \rtimes \mathcal{A}^{\circ}$. Recall that the algebra $\mathcal{A} \rtimes \mathcal{A}^{\circ}$ is the vector space $\mathcal{A} \otimes \mathcal{A}^{\circ}$ with product defined by

$$
(a \otimes f)(b \otimes g)=\left\langle f_{(1)}, b_{(2)}\right\rangle a b_{(1)} \otimes f_{(2)} g, \quad a, b \in \mathcal{A}, f, g \in \mathcal{A}^{\circ} .
$$

There is a left action of the algebra $\mathcal{A} \rtimes \mathcal{A}^{\circ}$ on $\mathcal{A}$ given by $f \triangleright b=\left\langle f, b_{(2)}\right\rangle b_{(1)}$ and $a \triangleright b=$ $a \cdot b$ for $f \in \mathcal{A}^{\circ}$ and $a, b \in \mathcal{A}$. Therefore, by matrix multiplication the matrix algebra $M_{n+1}\left(\mathcal{A} \rtimes \mathcal{A}^{\circ}\right)$ becomes a subalgebra of $L\left(\mathcal{A}_{n+1}\right)$, where $\mathcal{A}_{n+1}$ denotes the direct sum of $n+1$ copies of the algebra $\mathcal{A}$.

We define elements $C, \Omega_{k} \in M_{n+1}\left(\mathcal{A} \rtimes \mathcal{A}^{\circ}\right) \subseteq L\left(\mathcal{A}_{n+1}\right)$ by

$$
C=\left(\begin{array}{cccc}
0 & X_{1} & \cdots & X_{n} \\
X_{1} & 0 & \cdots & 0 \\
\vdots & \vdots & & \vdots \\
X_{n} & 0 & \cdots & 0
\end{array}\right), \quad \Omega_{k}=i\left(\begin{array}{cccc}
0 & f_{1}^{k} & \cdots & f_{n}^{k} \\
f_{1}^{k} & 0 & \cdots & 0 \\
\vdots & \vdots & & \vdots \\
f_{n}^{k} & 0 & \cdots & 0
\end{array}\right)
$$

and a homomorphism $\rho: \mathcal{A} \rightarrow L\left(\mathcal{A}_{n+1}\right)$ by $\rho(a)\left(b_{0}, \ldots, b_{n}\right)=\left(a b_{0}, \ldots, a b_{n}\right)$. Then the pair $(C, \rho)$ is a faithful algebraic commutator representation of the FODC $\Gamma$.

Now suppose in addition that $\mathcal{A}$ is a Hopf $*$-algebra and $\Gamma$ is a $*$-calculus. Then the cross product algebra $\mathcal{A} \rtimes \mathcal{A}^{\circ}$ is a *-algebra with involution determined by the involutions of $\mathcal{A}$ and $\mathcal{A}^{\circ}$. Let $\mathcal{A} \rtimes \mathcal{U}$ denote the *-subalgebra of $\mathcal{A} \rtimes \mathcal{A}^{\circ}$ generated by $\mathcal{A}$ and the functionals $X_{k}, f_{k}^{j}, j, k=1, \ldots, n$. Since $\Gamma$ is a $*$-calculus, $\mathcal{T}_{\Gamma}$ is $*$-invariant and we can assume without boss of generality that $X_{k}^{*}=X_{k}, k=1, \ldots, n$. Then $C$ is a hermitean element of $M_{n+1}(\mathcal{A} \rtimes \mathcal{U})$.

Let $\pi$ be a $*$-representation of the $*$-algebra $\mathcal{A} \rtimes \mathcal{U}$ on a domain $\mathcal{D}$. It gives rise in canonical way to $*$-representations, denoted again by $\pi$, of the $*$-algebras $M_{n+1}(\mathcal{A} \rtimes \mathcal{U})$ and $\mathcal{A}$ on $\mathcal{D}_{n+1}:=\mathcal{D} \oplus \ldots \oplus \mathcal{D}(n+1$ times $)$. Since $C$ is a hermitean element of 
$M_{n+1}(\mathcal{A} \rtimes \mathcal{U})$, the operator $D:=\pi(C)$ is symmetric on $\mathcal{D}_{n+1}$. Hence the pair $(D, \pi)$ is a commutator representation of the $*$-calculus $\Gamma$. The role of the left-invariant one-form $\omega_{k}$ in this commutator representation is played by the operator $\pi\left(\Omega_{k}\right)$. Since the matrix $\Omega_{k}$ contains the functionals $f_{j}^{k}, j, k=1, \ldots, n$, it is clear why unbounded commutators occur: For $*$-representations $\pi$ of $\mathcal{A} \rtimes \mathcal{U}$ the operators $\pi\left(f_{j}^{k}\right)$ are unbounded in general.

Suppose now that $\mathcal{A}$ is a compact quantum group algebra $(C Q G$ algebra), i.e. $\mathcal{A}$ is a Hopf $*$-algebra which is the span of matrix elements of finite dimensional unitary corepresentations of $\mathcal{A}$. Let $h$ be the Haar state of $\mathcal{A}$. There is a unique *-representation $\pi_{h}$ of $\mathcal{A} \rtimes \mathcal{A}^{\circ}$ such that its restriction to $\mathcal{A}$ is the GNS representation of the state $h$ with cyclic vector $\varphi_{h}$ and $\pi_{h}(f) \varphi_{h}=f(1) \varphi_{h}$ for $f \in \mathcal{A}^{\circ}$. From the preceding considerations we obtain

Proposition 2. For each finite dimensional first order *-calculus $\Gamma$ on a CQG algebra $\mathcal{A}$, the pair $\left(D, \pi_{h}\right)$ is a faithful commutator representation of $\Gamma$.

A. Jaffe [Ja] has developed a quantum harmonic analysis by allowing unbounded commutators $d a:=[D, \rho(a)]$. His crucial assumption is the boundedness of the operators $\left(I+D^{2}\right)^{-\beta / 2} d a\left(I+D^{2}\right)^{-\alpha / 2}$, where $\alpha, \beta \geq 0$ and $\alpha+\beta<1$. It can be shown that for the commutator representation $\left(C, \pi_{h}\right)$ described above of each of the $*$-calculi $\Gamma_{j}, j=1,2,5$, on $\mathcal{O}\left(S U_{q}(2)\right)$ Jaffe's boundedness condition is only satisfied if $\alpha+\beta \geq 1$. But in this case Jaffe's theory does not apply.

\section{Bicovariant first order differential calculi on coquasitriangular Hopf al-} gebras. In this section $\mathcal{A}$ is a coquasitriangular Hopf algebra (see e.g. [KS], 10.1).

Fix a universal $r$-form $\mathbf{r}$ of $\mathcal{A}$. Let $v=\left(v_{k}^{j}\right)_{j, k=1, \ldots, n}$ be a matrix corepresentation of $\mathcal{A}$ and let $\zeta$ be a character of $\mathcal{A}$ which is central in $\mathcal{A}^{\circ}$. As shown in [KS], Section 14.5, there is a bicovariant first order calculus $\Gamma_{v, \zeta}$ on $\mathcal{A}$ associated with $v$ and $\zeta$. This method for the construction of bicovariant FODC was first used in a very special case by Jurčo [Ju]. The structure of $\Gamma_{v, \zeta}$ is developed in detail in $[\mathrm{KS}]$. In this article we only need the fact that the quantum tangent space $\mathcal{T}_{v, \zeta}$ of $\Gamma_{v, \zeta}$ is the linear span of functionals $X_{k j}=\zeta l_{j}^{k}-\delta_{k j} \varepsilon, j, k=1, \ldots, n$, where $l_{j}^{k}$ are the $L$-functionals

$$
l_{j}^{k}(a)=\sum_{t} \mathbf{r}\left(v_{t}^{k} \otimes a_{(1)}\right) \mathbf{r}\left(a_{(2)} \otimes v_{j}^{t}\right) .
$$

By Proposition 10.16(ii) in [KS], the functional

$$
C_{v, \zeta}:=\sum_{i, j, k} X_{k j} \mathbf{r}\left(S^{2}\left(v_{i}^{j}\right) \otimes v_{k}^{j}\right)
$$

of $\mathcal{T}_{v, \zeta}$ belongs to the center of the Hopf dual $\mathcal{A}^{\circ}$. As in the preceding section, we consider $C_{v, \zeta}$ as an element of the cross product algebra $\mathcal{A} \rtimes \mathcal{A}^{\circ}$ acting on $\mathcal{A}$. Let $\rho: \mathcal{A} \rightarrow L(\mathcal{A})$ be defined by $\rho(a) b=a \cdot b, a, b \in \mathcal{A}$. The proofs of the next two results are given in [S2].

Proposition 3. The pair $\left(C_{v, \zeta}, \rho\right)$ is an algebraic commutator representation of the bicovariant FODC $\Gamma_{v, \rho}$.

Now let $\mathcal{A}$ be the coordinate Hopf algebra $\mathcal{O}\left(G_{q}\right)$, where $G_{q}$ is one of the quantum groups $S L_{q}(n+1), O_{q}(n)$ or $S p_{q}(2 n)$. 
TheOREM 4. Suppose that $q \in \mathbb{C}$ is transcendental.

(i) Then the algebraic commutator representation $\left(C_{v, \zeta}, \rho\right)$ is faithful.

(ii) If $q$ is real, then the pair $\left(\pi_{h}\left(C_{v, \zeta}\right), \pi_{h}\right)$ is a faithful commutator representation of the bicovariant first order $*$-calculus on the $C Q G$ algebra $\mathcal{A}=\mathcal{O}\left(G_{q}\right)$ with respect to the compact real form of $G_{q}$.

Remarks. 1.) Clearly, the bicovariant FODC $\Gamma_{v, \zeta}$ has the algebraic commutator representation described in the preceding section. But Theorem 4(i) states the much stronger result that the FODC $\Gamma_{v, \zeta}$ can be faithfully realized as a commutator inside the algebra $\mathcal{A} \rtimes \mathcal{A}^{\circ}$ by the single central element $C_{v, \zeta}$ of the Hopf dual $\mathcal{A}^{\circ}$.

2.) Let $G_{q}$ be $S L_{q}(n+1)$ or $S p_{q}(2 n)$ and assume that $q$ is transcendental. Then, as proved in [HS] (see [BS] for a related result), each finite dimensional bicovariant FODC of the coquasitriangular Hopf algebra $\mathcal{O}\left(G_{q}\right)$ is a direct sum of FODC $\Gamma_{v, \zeta}$. Combining this result with Theorem 4 it follows that for each finite dimensional bicovariant FODC $\Gamma$ of $\mathcal{O}\left(G_{q}\right)$ there is a central element $C \in \mathcal{O}\left(G_{q}\right)^{\circ}$ such that $(C, \rho)$ is a faithful commutator representation of $\Gamma$.

5. Dirac operator on the quantum disc. Suppose $q$ is a real number such that $0<q<1$. The coordinate algebra of the quantum disc $[\mathrm{KL}]$ is the unital $*$-algebra $\mathcal{O}\left(D_{q}\right)$ with single generator $z$ and defining relation

$$
z^{*} z-q^{2} z z^{*}=1-q^{2}
$$

It has been studied extensively in a series of papers by L. Vaksman and S. Sinel'shchikov (see the collection $[\mathrm{V}])$. The $*$-algebra $\mathcal{O}\left(D_{q}\right)$ is a left $\mathcal{U}_{q}(s u(1,1))$-module $*$-algebra with left action determined by

$$
E \triangleright z=-q^{1 / 2} z^{2}, \quad F \triangleright z=q^{1 / 2}, \quad K \triangleright z=q^{2} z .
$$

There is a unique FODC $\Gamma$ on the algebra $\mathcal{O}\left(D_{q}\right)$ such that $\Gamma$ is covariant with respect to the $\mathcal{U}_{q}(s u(1,1))$-action and the differentials $\left\{d z, d z^{*}\right\}$ form a left module basis of $\Gamma$ :

$$
d z \cdot z=q^{2} z \cdot d z, \quad d z \cdot z^{*}=q^{-2} z^{*} \cdot d z, \quad d z^{*} \cdot z=q^{2} z \cdot d z^{*}, \quad d z^{*} \cdot z^{*}=q^{-2} z^{*} \cdot d z^{*} .
$$

Obviously, $\Gamma$ is a $*$-calculus. Define an element $C \in M_{2}\left(\mathcal{O}\left(D_{q}\right)\right)$ and an algebra homomorphism $\rho: \mathcal{O}\left(D_{q}\right) \rightarrow M_{2}\left(\mathcal{O}\left(D_{q}\right)\right)$ by

$$
C=\left(1-q^{2}\right)^{-1}\left(\begin{array}{cc}
0 & z \\
z^{*} & 0
\end{array}\right), \quad \rho(f)=\left(\begin{array}{cc}
f & 0 \\
0 & f
\end{array}\right) .
$$

Then the pair $(C, \rho)$ is a faithful algebraic commutator representation of $\Gamma$.

There is a faithful $*$-representation $\pi$ of $\mathcal{O}\left(D_{q}\right)$ acting on the standard basis $\left\{e_{n}\right\}$ of the Hilbert space $l_{2}(\mathbb{N})$ by

$$
\pi(z) e_{n}=\left(1-q^{2 n+2}\right)^{1 / 2} e_{n+1}, \quad \pi\left(z^{*}\right) e_{n}=\left(1-q^{2 n}\right)^{1 / 2} e_{n-1}, \quad n \in \mathbb{N}_{0},
$$

where $e_{-1}:=0$. As usual we extend $\pi$ to matrices and obtain a faithful commutator representation $(\pi(C), \pi)$ of the $*$-calculus $\Gamma$.

In order to develop an invariant integration and a Dirac operator on the quantum disc, we need a *-algebra of "compactly supported functions" on the quantum disc. For notational simplicity, let us identify $\pi(f)$ with $f$ and $[\pi(C), \pi(f)]$ with $d f$ for $f \in \mathcal{O}\left(D_{q}\right)$. 
Let $\tilde{\mathcal{O}}\left(D_{q}\right)$ be the $*$-algebra generated by the $*$-algebra $\mathcal{O}\left(D_{q}\right)$ acting on $l_{2}(\mathbb{N})$ and the rank one projection $f_{0}:=e_{0} \otimes e_{0}$ and let $\mathcal{O}_{c}\left(D_{q}\right)$ be the two-sided $*$-ideal of $\tilde{\mathcal{O}}\left(D_{q}\right)$ generated by $f_{0}$. It can be shown that the $\mathcal{U}_{q}(s u(1,1))$-action on $\mathcal{O}\left(D_{q}\right)$ extends to $\tilde{\mathcal{O}}\left(D_{q}\right)$ by setting

$$
E \triangleright f_{0}=\left(q^{2}-1\right)^{-1} q^{1 / 2} z f_{0}, \quad F \triangleright f_{0}=\left(1-q^{-2}\right)^{-1} q^{1 / 2} f_{0} z^{*}, \quad K^{ \pm 1} \triangleright f_{0}=f_{0}
$$

such that $\tilde{\mathcal{O}}\left(D_{q}\right)$ becomes a $\mathcal{U}_{q}(s u(1,1))$-module $*$-algebra. Then

$$
h(x):=\operatorname{Tr}\left(1-z^{*} z\right)^{-1} x, \quad x \in \mathcal{O}_{c}\left(D_{q}\right),
$$

is a $\mathcal{U}_{q}(s u(1,1))$-invariant faithful positive linear functional on the $*$-algebra $\mathcal{O}_{c}\left(D_{q}\right)$. Clearly, $h$ can be considered as a quantum analog of the $s u(1,1)$-invariant integration $\int(1-\bar{z} z)^{-1} \varphi d z d \bar{z}$ on the unit disc.

The FODC $\Gamma$ on $\mathcal{O}\left(D_{q}\right)$ is extended to a $\mathcal{U}_{q}(s u(1,1))$-covariant FODC $\tilde{\Gamma}$ on $\tilde{\mathcal{O}}\left(D_{q}\right)$ by defining $\tilde{d} x:=i[C, x], x \in \tilde{\mathcal{O}}\left(D_{q}\right)$. The corresponding universal higher order calculus on $\tilde{\mathcal{O}}\left(D_{q}\right)$ is denoted by $\tilde{\Gamma}^{\wedge}$. Its structure is very simple: The 2 -forms have the right module basis element $d z \wedge d z^{*}$, while for $k \geq 3$ all $k$-forms vanish. Let $\Gamma_{c}^{\wedge}$ denote the restriction of the differential calculus $\tilde{\Gamma}^{\wedge}$ to the algebra $\mathcal{O}_{c}\left(D_{q}\right)$.

Before proceeding we give the following general

Definition 5 . Let $\mathcal{X}$ be a left module $*$-algebra of a Hopf $*$-algebra $\mathcal{U}$ and let $\Gamma$ be a right $\mathcal{X}$-module. A covariant metric on $\Gamma$ is a sesquilinear mapping $g: \Gamma \times \Gamma \rightarrow \mathcal{X}$ such that:

(i) $g(\gamma, \gamma) \geq 0$ for all $\gamma \in \Gamma$ and $g(\gamma, \gamma)=0$ implies $\gamma=0$.

(ii) $g(\gamma x, \eta y)=y^{*} g(\gamma, \eta) x$ for $x, y \in \mathcal{X}$ and $\gamma, \eta \in \Gamma$.

(iii) $f \triangleright g(\gamma, \eta)=g\left(f_{(2)^{\triangleright}} \gamma, S\left(f_{(1)}\right)^{*} \triangleright \eta\right)$ for $f \in \mathcal{U}$ and $\gamma, \eta \in \Gamma$.

For the differential calculus $\Gamma_{c}^{\wedge}$ of $\mathcal{O}_{c}\left(D_{q}\right)$ there exists a covariant metric $g$ given by

$$
\begin{aligned}
& g\left(a_{0}+d z \cdot a_{1}+d z^{*} \cdot a_{2}+d z \wedge d z^{*} \cdot a_{3}, b_{0}+d z \cdot b_{1}+d z^{*} \cdot b_{2}+d z \wedge d z^{*} \cdot b_{3}\right) \\
& =b_{0}^{*} a_{0}+\frac{1}{1+q^{2}}\left(b_{1}^{*}\left(1-z z^{*}\right)^{2} a_{1}+b_{2}^{*}\left(1-z z^{*}\right)^{2} a_{2}\right)+\frac{1}{q^{2}\left(1+q^{2}\right)} b_{3}^{*}\left(1-z z^{*}\right)^{4} a_{3} .
\end{aligned}
$$

Then, $\langle\gamma, \eta\rangle:=h(g(\gamma, \eta)), \gamma, \eta \in \Gamma_{c}^{\wedge}$, defines a scalar product on $\Gamma_{c}^{\wedge}$. Let $\Gamma_{c}^{\wedge k}$ be the $k$-forms and let $d_{k}: \Gamma_{c}^{\wedge k} \rightarrow \Gamma_{c}^{\wedge(k+1)}$ be the differentiation of the differential calculus $\Gamma_{c}^{\wedge}$. Then there are linear operators $d_{k}^{*}: \Gamma_{c}^{\wedge(k+1)} \rightarrow \Gamma_{c}^{\wedge k}$ such that $\left\langle d_{k} \gamma, \eta\right\rangle=\left\langle\gamma, d_{k}^{*} \eta\right\rangle, \gamma \in$ $\Gamma_{c}^{\wedge k}, \eta \in \Gamma_{c}^{\wedge(k+1)}$. The operator

$$
D=\left(\begin{array}{ccc}
0 & d_{1}^{*} & 0 \\
d_{1} & 0 & d^{*} \\
0 & d_{2} & 0
\end{array}\right)
$$

on $\Gamma_{c}^{\wedge}=\Gamma_{c}^{\wedge 0} \oplus \Gamma_{c}^{\wedge 1} \oplus \Gamma_{c}^{\wedge 2}$ is called the Dirac operator of the quantum disc. The operator $D$ is bounded and its closure on the Hilbert space completion of $\Gamma_{c}^{\wedge}$ is self-adjoint. Explicit formulas for the operator $D$ are given in [SSV].

Set $\rho(x) \eta:=x \eta$ for $x \in \mathcal{O}_{c}\left(D_{q}\right)$ and $\eta \in \Gamma_{c}^{\wedge}$. Then the pair $(D, \rho)$ is a commutator representation of the first order $*$-calculus $\Gamma_{c}^{\wedge 1}$ of the algebra $\mathcal{O}_{c}\left(D_{q}\right)$.

6. Dirac operator on the real quantum quarter plane. Suppose $q$ is a complex number such that $|q|=1$ and $q \neq \pm 1$. Let $\mathcal{O}\left(\mathbb{R}_{q}^{2}\right)$ be the $*$-algebra with two hermitean 
generators $x$ and $y$ such that $x y=q y x$ and let $\hat{\mathcal{O}}\left(\mathbb{R}_{q}^{2}\right)$ be the $*$-algebra with hermitean generators $x, y, y^{-1}$ and relations $x y=q y x$ and $y y^{-1}=y^{-1} y=1$. We consider $\mathcal{O}\left(\mathbb{R}_{q}^{2}\right)$ as a *-subalgebra of $\hat{\mathcal{O}}\left(\mathbb{R}_{q}^{2}\right)$.

There are two distinguished first order differential $*$-calculi (invented in $[\mathrm{PW}]$ and [WZ]) of the $*$-algebra $\mathcal{O}\left(\mathbb{R}_{q}^{2}\right)$. One of these calculi, denoted by $\Gamma$ in what follows, has the relations

$$
\begin{array}{ll}
x \cdot d x=q^{-2} d x \cdot x, & y \cdot d x=q^{-1} d x \cdot y+\left(q^{-2}-1\right) d y \cdot x, \\
x \cdot d y=q^{-1} d y \cdot x, & y \cdot d y=q^{-2} d y \cdot y .
\end{array}
$$

Set $\hat{\mathcal{O}}\left(\mathbb{R}_{q}^{2}\right)_{2}:=\hat{\mathcal{O}}\left(\mathbb{R}_{q}^{2}\right) \oplus \hat{\mathcal{O}}\left(\mathbb{R}_{q}^{2}\right)$ and define an element

$$
C:=\left(q^{2} x^{2} y^{-2}, y^{-2}\right) \in \hat{\mathcal{O}}\left(\mathbb{R}_{q}^{2}\right)_{2}
$$

and a homomorphism $\rho: \mathcal{O}\left(\mathbb{R}_{q}^{2}\right) \rightarrow L\left(\hat{\mathcal{O}}\left(\mathbb{R}_{q}^{2}\right)_{2}\right)$ by $\rho(a)\left(b_{1}, b_{2}\right)=\left(a b_{1}, a b_{2}\right), a \in \mathcal{O}\left(\mathbb{R}_{q}^{2}\right)$, $b_{1}, b_{2} \in \hat{\mathcal{O}}\left(\mathbb{R}_{q}^{2}\right)$. Then the pair $(C, \rho)$ is a faithful algebraic commutator representation of $\Gamma$. Note that the element $C \in \hat{\mathcal{O}}\left(\mathbb{R}_{q}^{2}\right)_{2}$ is hermitean.

Fix $\alpha, \beta \in \mathbb{R}$ such that $q=e^{2 \pi i \alpha \beta}$. Let $\mathcal{P}$ and $\mathcal{Q}$ be the self-adjoint operators on the Hilbert space $L^{2}(\mathbb{R})$ given by $\mathcal{P} f=(2 \pi i)^{-1} f^{\prime}$ and $\mathcal{Q} f=t f(t)$. There is a *-representation $\pi$ of $\mathcal{O}\left(\mathbb{R}_{q}^{2}\right)$ on $L^{2}(\mathbb{R})$ such that $\pi(x)=e^{2 \pi \alpha Q}$ and $\pi(y)=e^{2 \pi \beta \mathcal{P}}$. Then the pair $(\pi(C), \pi)$ is a faithful commutator representation of the $*$-calculus $\Gamma$. For simplicity, we write $f$ for $\pi(f)$.

In order to develop quantum analogs of the Lebesgue integration and of a $*$-algebra of integrable $C^{\infty}$-functions, we use the Weyl calculus of pseudodifferential operators. Let $\mathfrak{A}\left(\mathbb{R}^{2}\right)$ be the set of holomorphic functions on $\mathbb{C}^{2}$ such that

$$
\sup _{\left|y_{j}\right| \leq k} \iint\left|a\left(x_{1}+i y_{1}, x_{2}+i y_{2}\right)\right|^{2} e^{k\left(\left|x_{1}\right|+\left|x_{2}\right|\right)} d x_{1} d x_{2}<\infty
$$

for all $k \in \mathbb{N}$. Each $a \in \mathfrak{A}\left(\mathbb{R}^{2}\right)$ acts as an operator on the dense domain $\mathcal{D}:=\cap_{n, m=1}^{\infty} \mathcal{D}\left(x^{n} y^{m}\right)$ of $L^{2}(\mathbb{R})$ by

$$
(a \varphi)(t)=\iint a\left(\frac{1}{2}(t+s), x\right) e^{2 \pi i(t-s) x} \varphi(s) d s d x, \quad \varphi \in \mathcal{D} .
$$

The direct sum $\mathcal{A}\left(\mathbb{R}_{q}^{++}\right):=\mathcal{O}\left(\mathbb{R}_{q}^{2}\right) \oplus \mathfrak{A}\left(\mathbb{R}^{2}\right)$ is a $*$-algebra equipped with the operator product and involution on $L^{2}(\mathbb{R})$ (see [S3] for explicit formulas). We think of elements of $\mathcal{A}\left(\mathbb{R}_{q}^{++}\right)$as "functions on the quantum quarter plane" $\mathbb{R}_{q}^{++}$, because the spectra of the two coordinate functions $x$ and $y$ are the non-negative half-line $[0,+\infty)$.

Further, $\mathcal{O}\left(\mathbb{R}_{q}^{2}\right)$ is a left $U_{q}\left(s l_{2}(\mathbb{R})\right)$-module $*$-algebra with left action determined by

$$
E \triangleright x=y, \quad E \triangleright y=0, \quad F \triangleright x=0, \quad F \triangleright y=x, \quad K \triangleright x=q^{-1 / 2} x, \quad K \triangleright y=q^{1 / 2} y .
$$

This action extends to an action on the larger algebra $\mathcal{A}\left(R_{q}^{++}\right)$such that $\mathcal{A}\left(\mathbb{R}_{q}^{++}\right)$becomes a left $U_{q}\left(s l_{2}(\mathbb{R})\right)$-module $*$-algebra. The generators $E, F, K$ of $U_{q}\left(\operatorname{sl}_{2}(\mathbb{R})\right)$ act on $a \in$ $\mathfrak{A}\left(\mathbb{R}^{2}\right)$ by

$$
\begin{aligned}
& (E \triangleright a)\left(x_{1}, x_{2}\right)=\lambda^{-1} e^{2 \pi\left(\beta x_{2}-\alpha x_{1}\right)}\left(a\left(x_{1}+\beta i, x_{2}\right)-a\left(x_{1}-\beta i, x_{2}\right)\right), \\
& (F \triangleright a)\left(x_{1}, x_{2}\right)=\lambda^{-1} e^{2 \pi\left(\alpha x_{1}-\beta x_{2}\right)}\left(a\left(x_{1}, x_{2}+\alpha i\right)-a\left(x_{1}, x_{2}-\alpha i\right)\right), \\
& (K \triangleright a)\left(x_{1}, x_{2}\right)=a\left(x_{1}-\frac{\beta}{2} i, x_{2}+\frac{\alpha}{2} i\right) .
\end{aligned}
$$


The linear functional $h$ on the $*$-algebra $\mathfrak{A}\left(\mathbb{R}^{2}\right)$ defined by

$$
h(a):=\iint e^{2 \pi\left(\alpha x_{1}+\beta x_{2}\right)} a\left(x_{1}, x_{2}\right) d x_{1} d x_{2}, \quad a \in \mathfrak{A}\left(\mathbb{R}^{2}\right),
$$

is $\mathcal{U}_{q}\left(\operatorname{sl}_{2}(\mathbb{R})\right)$-invariant, faithful and positive. It plays the role of the invariant integration of the quantum quarter plane.

The FODC $\Gamma$ on $\mathcal{O}\left(\mathbb{R}_{q}^{2}\right)$ is $U_{q}\left(s l_{2}(\mathbb{R})\right)$-covariant. We use the commutator representation of $\Gamma$ obtained above to extend the calculus to the larger algebra $\mathcal{A}\left(\mathbb{R}_{q}^{++}\right)$. That is, for $z \in \mathcal{A}\left(\mathbb{R}_{q}^{++}\right)$we define an FODC $\tilde{\Gamma}$ of $\mathcal{A}\left(\mathbb{R}_{q}^{++}\right)$by setting $\tilde{d} z:=i[C, z]$. The differentials $\{\tilde{d} x, \tilde{d} y\}$ form a right module basis of $\tilde{\Gamma}$, so for each $z \in \mathcal{A}\left(\mathbb{R}_{d}^{++}\right)$there are uniquely determined elements $\partial_{x}(z), \partial_{y}(z) \in \mathcal{A}\left(\mathbb{R}_{q}^{++}\right)$such that

$$
\tilde{d} z=\tilde{d} x \cdot \partial_{x}(z)+\tilde{d} y \cdot \partial_{y}(z) .
$$

For $z=a \in \mathfrak{A}\left(\mathbb{R}_{q}^{2}\right)$ the partial derivatives $\partial_{x}(a), \partial_{y}(a)$ are given by

$$
\begin{aligned}
& \partial_{x}(a)=\left(1-q^{-2}\right)^{-1} e^{-2 \pi \alpha x_{1}}\left(a\left(x_{1}, x_{2}-\frac{\alpha}{2} i\right)-a\left(x_{1}-2 \beta i, x_{2}-\frac{\alpha}{2} i\right)\right), \\
& \partial_{y}(a)=\left(1-q^{-2}\right)^{-1} e^{-2 \pi \beta x_{2}}\left(a\left(x_{1}-\frac{3}{2} \beta i, x_{2}\right)-a\left(x_{1}-\frac{3}{2} \beta i, x_{2}-2 \alpha i\right)\right) .
\end{aligned}
$$

Let $\Gamma_{c}$ denote the restriction of $\tilde{\Gamma}$ to the subalgebra $\mathfrak{A}\left(\mathbb{R}^{2}\right)$ and let $\Gamma_{c}^{\wedge}$ be the corresponding higher order calculus of $\mathfrak{A}\left(\mathbb{R}^{2}\right)$. For the calculus $\Gamma_{c}^{\wedge}$ on $\mathfrak{A}\left(\mathbb{R}^{2}\right)$ there exists a covariant metric $g$ defined by

$$
\begin{aligned}
& g\left(a_{0}+d x \cdot a_{1}+d y \cdot a_{2}+d x \wedge d y \cdot a_{3}, b_{0}+d x \cdot b_{1}+d y \cdot b_{2}+d x \wedge d y \cdot b_{3}\right) \\
& =b_{0}^{*} a_{0}+b_{1}^{*} x^{2} a_{1}+b_{2}^{*} y^{2} a_{2}+b_{2}^{*} y x a_{1}+b_{1}^{*} x y a_{2}+b_{3}^{*} y x^{2} y a_{3} .
\end{aligned}
$$

Now we proceed verbatim as in the preceding section and obtain a Dirac operator $D$ on the quantum quarter plane. Letting $\rho(a) \eta=a \eta$ for $a \in \mathfrak{A}\left(\mathbb{R}^{2}\right)$, the pair $(D, \rho)$ is a commutator representation of the first order $*$-calculus of the algebra $\mathfrak{A}\left(\mathbb{R}^{2}\right)$.

\section{References}

[BS] P. Baumann and F. Schmitt, Classification of bicovariant differential calculi on quantum groups, Commun. Math. Phys. 194 (1998), 71-86.

[C] A. Connes, Non-commutative Geometry. Academic Press, New York, 1994.

[H1] I. Heckenberger, Spin geometry on quantum groups via covariant different calculi, Adv. Math. 175 (2003), 197-242.

[H2] I. Heckenberger, Classification of left-covariant differential calculi on the quantum group $S L_{q}(2)$, J. Algebra 237 (2001), 203-237.

[HS] I. Heckenberger and K. Schmüdgen, Classification of bicovariant differential calculi on the quantum groups $S L_{q}(n+1)$ and $S p_{q}(2 n)$, J. Reine Angew. Math. 502 (1998), 141-162.

[Ja] A. Jaffe, Quantum harmonic analysis and geometric invariants, Adv. Math. 143 (1999), $1-110$.

[JL] A. Joseph and G. Letzter, Local finiteness of the adjoint action for quantized enveloping algebras, J. Algebra 153 (1992), 289-318.

[Ju] B. Jurčo, Differential calculus on quantized simple Lie groups, Lett. Math. Phys. 22 (1991), 177-186. 
[KL] S. Klimek and A. Lesniewski, A two-parameter quantum deformation of the unit disc, J. Funct. Anal. 115 (1993), 1-23.

[KMT] J. Kustermans, G. J. Murphy and L. Tuset, Differential calculi over quantum groups and twisted cyclic cocycles, J. Geom. Phys. 44 (2003), 570-594.

[KS] A. Klimyk and K. Schmüdgen, Quantum Groups and Their Representations, SpringerVerlag, Heidelberg, 1997.

[PS] M. Paschke and A. Sitarz, The geometry of noncommutative symmetries, Acta Phys. Pol. B 31 (2000), 1897-1911.

[PW] W. Pusz and S. L. Woronowicz, Twisted second quantization, Rep. Math. Phys. 27 (1989), 231-257.

[S1] K. Schmüdgen, Commutator representations of differential calculi on the quantum group $S U_{q}(2)$, J. Geom. Phys. 31 (1999), 241-264.

[S2] K. Schmüdgen, Commutator representations of covariant differential calculi on quantum groups, Lett. Math. Phys. 59 (2002), 95-106.

[S3] K. Schmüdgen, The quantum quarter plane and the real quantum plane, Internat. J. Math. 13 (2002), 279-321.

[SSV] S. Sinel'shchikov, K. Schmüdgen and L. Vaksman, The Dirac operator and the Clifford algebra of the quantum disc, article in [V].

[V] L. Vaksman, Lectures on q-analogues of Cartan domains and associated HarishChandra modules, math.QA /0109198.

[WZ] J. Wess and B. Zumino, Covariant differential calculus on the quantum hyperplane, Nucl. Phys. B Proc. Suppl. 18 (1991), 302-312.

[W] S. L. Woronowicz, Differential calculus on quantum matrix pseudogroups (quantum groups), Commun. Math. Phys. 122 (1989), 125-170. 